\title{
A Study on the Effect of Feedback on the Motor Learning of Adjustability of Grasping Force in the Elderly
}

Ryohei Yamamoto ( $\nabla$ r-yamamoto@kyushu-ns.ac.jp )

Kyushu University of Nursing and Social Welfare

Kazunori Akizuki

Kobe International University

Kazuto Yamaguchi

Ibaraki Prefectural University of Health Sciences

Jun Yabuki

Ibaraki Prefectural University of Health Sciences Hospital

Tatsuya Kaneno

Mejiro University

Research Article

Keywords: feedback, motor learning, grasping force, AGF

Posted Date: December 15th, 2021

DOI: https://doi.org/10.21203/rs.3.rs-1124029/v1

License: (c) (1) This work is licensed under a Creative Commons Attribution 4.0 International License. Read Full License 


\section{Abstract}

In this study, we investigated the function related to the adjustability of grasping force (AGF) of elderly people and the effectiveness of concurrent visual feedback in learning tasks that require AGF. The young and elderly groups were measured for simple visual reaction time and a $100 \mathrm{~g}$ AGF task that reflected the difference between desired performance and actual performance, and the main learning task was tested and practiced using concurrent visual feedback. Performance improved from pre-test to retention test in both groups, but the elderly group failed to reach the level of the young group. In addition, the retention test performance was associated with age and pre-test performance. In the elderly group, the initial performance of the practice was associated with the difference between desired performance and actual performance. In the latter half of the exercise, it was associated with visual-motor speed. The results of this study show that concurrent visual feedback is effective for learning tasks that require AGF. Indicatively, the improvement in performance during practice is insufficient, and the learning effect is reduced in elderly people who have a significant difference between desired performance and actual performance or whose visual-motor speed is slow.

\section{Introduction}

In activities of daily living, it is necessary to grasp the object with an appropriate force according to the shape and weight of the object, and fine adjustment of the force is required. Previous studies have defined this ability as the adjustability of grasping force (AGF) and reported that elderly people showed lower AGF than young people[1, 2]. By establishing a practice method that promotes the acquisition of tasks that require AGF in the elderly, improvement in the degree of independence in the daily life of the elderly is expected. Motor learning is the process of learning some kind of movement from experience, such as practice and holding it for a long period of time. Raz et al.[3] reported that older people had lower motor performance than younger people when using a one-handed position adjustment task called the pursuit rotor task, which tracks a metal stylus against a moving target. Rodrigue et al.[4] also reported that the elderly required more practice to reach the same level of performance in upper limb mirror tracing tasks as young people. Established from these reports, the performance of the elderly with regard to their accuracy and speed of movements is lower than that of the young, and it takes a lot of time to improve the performance.

Visual feedback is a technique used in various practice settings, such as rehabilitation and sports. Effectively obtaining information related to a task is important for learning new movements[5]. Visual feedback is classified into two types, concurrent feedback and terminal feedback, depending on the timing of feedback, and the effect differs depending on the difficulty of the learning task[6]. Concurrent feedback given in parallel with movement improves performance during practice in simple tasks but degrades motor learning[7-9]. Contrarily, a comparison of cognitive load aspects reveals that concurrent feedback has less cognitive load than terminal feedback[10] and has been reported to be effective for learning complex tasks that tend to increase cognitive load[11, 12]. Thus, the effects of feedback on motor learning change depending not only on the complexity of the task but also on how difficult the task 
is for the learner. Terminal feedback is effective when the complexity of the task is low for the learner, while concurrent feedback is effective when the complexity is high[13]. When these are applied to the elderly, it is presumed that the same task will be more difficult for the elderly with various deteriorations in mental and physical functions than younger individuals. Wishart et al. examined the effects of concurrent and terminal feedback for motor learning on complex upper limb coordination tasks in young and elderly people[14]. The results demonstrated that motor learning occurred in both concurrent and terminal feedback in young people, but motor learning occurred only in the practice of concurrent feedback in the elderly. In addition, performance improvement and motor learning occurred later in the elderly than in young people. Thus, especially in the early stages of practice for the elderly, using concurrent visual feedback is effective for motor learning. However, it is thought that the effect will be lower than that for young people.

A study on AGF reported that the AGF of the elderly was lower than that of the young using a method of approximating the grasping force to the target value displayed on the screen[1]. A previous study has reported that the AGF of elderly people is lower than that of young people under concurrent visual feedback conditions, in which the grasping force is adjusted according to the visual information displayed on the monitor[1]. This method is similar to practice using concurrent feedback, and the performance is expected to be influenced by the speed at which visual information is reflected in movement and the ability to properly adjust the grasping force without relying on the other senses. The speed at which visual information is reflected in movement is called visual-motor speed and is measured using a simple visual reaction time (VRT) in a computer-based neurobehavioural evaluation system, which is a computer-based test[15]. Many studies have reported that the simple visual reaction time increases with age[16-18]. In addition, the function of appropriately adjusting competence without relying on other senses is considered to be the difference between the desired and actual performance. Reportedly, ageing causes a decrease in functions such as reaction speed and accuracy of movements, and the degree of this difference is likely to increase[19]. However, the association of these functional declines with the performance of AGF tasks using visual information in the elderly has not been investigated. In addition, it has not been examined whether practice using concurrent visual feedback, which is effective for motor learning in the elderly using other learning tasks, is effective for learning AGF tasks. In addition, the effectiveness of practice with concurrent visual feedback, which has been reported to be effective for motor learning in the elderly, has not been examined for the learning of tasks that require AGF.

In this study, we investigated the effect of practice using concurrent visual feedback on the learning of the AGF task in elderly people. Furthermore, we investigated how the visual-motor speed and the degree of difference between the desired performance and actual performance are related to the AGF. Our first hypothesis is that practice with concurrent visual feedback is useful for learning the AGF task in older people. However, older people do not achieve the same performance as younger people. The second hypothesis is that the performance of the AGF task is affected by the visual-motor speed, which decreases with ageing and the difference between the desired performance and actual performance. 


\section{Methods}

\section{Participants}

The eligibility criteria for the young participants included in the study were $20 \leq$ age $<30$ years and were affiliated with the Faculty of Nursing and Welfare, the Kyushu University of Nursing and Social Welfare, in Japan. The exclusion criteria included having an experience of carrying out tasks similar to those required to be performed for this study or having a current or past (history of) orthopaedic or neurological disease of the hands and fingers that impacted ADL. The eligibility criteria for elderly individuals were age $\geq 65$ years, those living in their own homes (main place of residence), and able to ambulate without a walking aid, and able to travel to the study venue by themselves. The exclusion criteria for the latter group were having an experience of carrying out tasks similar to those required for this study, having a current or past (history of) an orthopaedic or neurological disease of the hands and fingers that impacted ADL, or scoring $\leq 23$ on the Mini-Mental State Examination.

A power analysis was conducted to estimate the sample size using $G *$ Power 3.1.9.7. The sample size calculation was considered as a power calculation to detect differences between the groups in the performance of a task that required AGF during the test sessions. We used repeated measured analysis of variance (ANOVA), within-between interaction with an a error level probability of 0.05 , and a power (1- $\beta$ error probability) of $80 \%$. The medium effect size Cohen was set to $f=0.25$. The analysis described above revealed that a total sample size of 34 was required for this study. Therefore, recruitment was closed when 17 applicants from each group were confirmed. As a result, 36 healthy individuals ( 28 men and 8 women) participated in this study. The participants were divided into two age groups. The young group ( 14 women, 4 men) were aged between 20 and 22 years (mean age $=21, S D=0.3$ ) and the elderly group ( 14 women, 4 men) were aged between 60 and 75 years (mean age $=71, S D=3.9$ ).

None of the participants reported any neurological or vestibular disorders or orthopaedic conditions before participating in the study. The participants had no prior experience with the learning task, and they were not informed of the specific purpose of our study. A preliminary explanation of the study details was provided to all participants, and written consent was obtained. Our study protocol was approved by the Institutional Review Board of Kobe International University (G2019-102). A preliminary explanation of the study details was provided to all participants, and written informed consent was obtained. All procedures were approved by the institutional review board and performed in accordance with the Declaration of Helsinki.

\section{Equipment and tasks}

In this study, iWakka (Aimu Co., Ltd.) was used to measure AGF based on a report by Kaneno ${ }^{[1,2]}$ (Fig. 1). Written informed consent for publication of an image of Fig. 1 was obtained from one of the authors. iWakka is a cylindrical device with a height of $80 \mathrm{~mm}$ and a diameter of $65 \mathrm{~mm}$ and can measure a grasping force of $0-400 \mathrm{~g}$ depending on the degree of opening and closing of the device. During the 
measurement, the subject held the iWakka in a sitting position with one hand and continuously adjusted the grasping force to approximate an arbitrarily set target value. The measured value and the target value during measurement can be displayed as feedback on a monitor placed in front of the participant. Since

the AGF is calculated as the root mean square error (RMSE) from the absolute error per unit time between the measured value and the target value, a smaller absolute value is indicative of a better AGF.

Two AGF tasks were used in the study. First, a 100g AGF task was set as an index of difference between the desired and actual performance in the state, excluding the visual information and the temporal aspect. In this task, the participant was asked to adjust the measurement value of iWakka to $100 \mathrm{~g}$ for $10 \mathrm{~s}$ without looking at the monitor. Next, the main learning task was set as a task to be learned through practice. In this task, the participants were asked to continuously adjust their grasping force to match the target values, as shown in Fig. 2. The task consisted of 30 seconds per trial.

\section{Visual-Motor Speed}

In this study, referring to the report by Cuthbertson et al. ${ }^{[20]}$, visual reaction time (VRT) was measured as an index reflecting visual-motor speed using the website www.humanbenchmark.com. We performed this task using a computer that could connect to the Internet. The participants were asked to click the mouse as soon as possible when the colour of the front screen automatically changed from red to green. The time (milliseconds) from when the screen turns green to when the participant clicks the mouse is automatically calculated.

\section{Procedure}

The elderly and young groups were measured for 2 days using the same procedure (Fig. 3). Prior to the measurement, the dominant hand of each participant was determined using the Edinburgh Handedness Scale. In this study, the learners performed all the tasks with their non-dominant hands. As a result of the Edinburgh Handedness Scale, all the participants were right-handed, so they performed the task with their left hand.

After determining the dominant hand, the $100 \mathrm{~g}$ AGF task was measured five times. No feedback was given to prevent the participant from correcting the difference between the desired performance and actual performance. After the completion of the $100 \mathrm{~g}$ AGF task, the visual simple reaction time was measured five times.

Subsequently, the participants performed a pre-test, acquisition phase, and retention test of the main learning task. In the pre-test, the participants performed four trials of the task without feedback. The acquisition phase consisted of three blocks with one block in four trials. Subsequently, the participants performed a total of 12 trials. During the acquisition phase, the participant was given concurrent feedback by confirming the target value and the actual measured value displayed on the monitor. A 20second break was set between each trial, and a 60 -second break was set between each block. The 
retention test was conducted $24 \mathrm{~h}$ after completion of the acquisition phase with similar contents to the pre-test.

\section{Outcome measures and statistical analysis}

In this study, the RMSE of the $100 \mathrm{~g}$ AGF task and the main learning task were used as parameters of the AGF. For the 100g AGF task, the RMSE was calculated from the target value and the measured value with 5 to 10 s of the measurement time as the analysis range, and the average value of five trials was calculated. Next, in the main learning task, the RMSE was calculated with a measurement time of 5 to 30 s as the analysis range, and the average value was calculated in the pre-test, each block of the acquisition phase, and the retention test.

Statistical analyses were conducted using IBM SPSS Statistics 25 (IBM Corp., NY, USA) for Windows. The design for the analysis of the test and acquisition data was a $2 \times 2$ (generation $\times$ test) ANOVA with repeated measures on the last factor. When a significant main effect or interaction was obtained, the paired sample t-test and the independent sample t-test were performed as post hoc tests. Independent sample t-tests were also performed on the $100 \mathrm{~g}$ AGF task and VRT in the elderly and young groups.

In addition, to examine factors related to motor learning of the tasks that require AGF, a multivariable linear regression analysis using the stepwise method was performed for all participants. The analysis used the RMSE of the retention test as the objective variable, the age, and the RMSE of the pretest as the explanatory variables. Furthermore, to analyse the difference between desired performance and actual performance and the effect of visual-motor speed on performance during the acquisition phase, a multivariable linear regression analysis was performed on each group of participants. In this analysis, a stepwise method was used with the RMSE of each block in the acquisition phase as the objective variable and the $100 \mathrm{~g}$ AGF task and VRT as the explanatory variables. Statistical significance was set at $p<0.05$.

\section{Results}

\section{RMSE of the main learning task in the pretest and retention test}

A two-way ANOVA of $2 \times 2$ (generation $\times$ test) was performed with the RMSE of each test as the objective variable for the elderly and young groups (Fig. 4a). A significant main effect was found for the factors within the participant $\left(F(1,34)=7.48, p<0.05, n p^{2}=0.180\right)$. In addition, a significant main effect was observed for the factors between participants $\left(F(1,34)=5.73, p<0.05, \eta p^{2}=0.144\right)$. No significant interaction was observed. As a post hoc test after the two-way ANOVA, we performed an independent sample t-test with the RMSE of the main learning task as the objective variable for the elderly and young groups (Fig. 4b). As a result, the young group showed significantly smaller values than the elderly group $(p<0.001$, difference in mean 3.99, 95\% Cl [1.06330, 6.92059], Cohen's $d=0.42)$. In addition, paired t-tests were performed for the RMSE of the pre-test and retention test for all participants (Fig. 4c). As a result, the 
retention test showed a significantly smaller value than the pre-test $(p<0.01$, difference in mean $6.61,95 \%$ Cl [2.19570, 11.02643], Cohen's d = 0.72).

\section{The $100 \mathrm{~g}$ AGF task}

Independent sample t-tests were performed on the elderly and young groups, with the RMSE of the $100 \mathrm{~g}$ AGF task as the objective variable (Fig. 4d). No significant difference was observed between the groups. However, a moderate effect size was observed ( $p=0.061$, difference in mean $0.06,95 \% \mathrm{Cl}[-0.00313,0.13]$, Cohen's $d=0.66$ ).

\section{Visual-Motor Speed}

Independent sample t-tests were performed on the elderly and young groups using VRT as the objective variable (Fig. 4e). As a result, the young group showed significantly smaller values than the elderly group ( $p<0.001$, difference in mean 73.56, 95\% Cl [37.28644, 109.82], Cohen's $d=1.51$ ).

\section{The multivariable linear regression analysis of RMSE of the retention test}

Multivariable linear regression analysis was performed with the RMSE of the retention test in all participants as the objective variable and the RMSE of the pre-test and age as the explanatory variables (Table 1). As a result, a model of RMSE of the retention test $=0.273+0.388 \times$ the RMSE of the pretest + $0.97 \times$ age was calculated $\left(R=0.689, R^{2}=0.474, p<0.001\right)$.

Table 1

Multivariable linear regression analysis of retention test performance for all participants

\begin{tabular}{|clll|}
\hline RMSE of Retention test & SE $\boldsymbol{\beta}(95 \% \mathrm{Cl})$ & B & p-Value \\
\hline Retention test & & & \\
\hline RMSE of Pretest & $0.533(0.193-0.583)$ & 0.388 & 0.000 \\
\hline AGE & $0.312(0.014-0.181)$ & 0.097 & 0.024 \\
\hline R $^{2}$ & 0.474 & & \\
\hline F & 14.894 & & \\
\hline SE $\beta$ : standardised coefficient; Cl: confidence interval, RMSE: Root Mean Square Error \\
\hline
\end{tabular}

\section{The multivariable linear regression analysis of RMSE of the acquisition phase}

Multivariable linear regression analysis was performed with RMSE as the objective variable and the $100 \mathrm{~g}$ AGF task and VRT as explanatory variables in each block of the acquisition phase (Table 2). As a result, the following model was calculated for each block in the elderly group: First, in block 1, a model called RMSE $=2.598+18.571 \times 100 \mathrm{~g}$ AGF task was calculated $\left(R=0.639, R^{2}=0.408, p<0.01\right)$. In block 2 , the 
model of RMSE $=-0.195+0.003 \times$ VRT was calculated $\left(R=0.495, R^{2}=0.245, p<0.05\right)$. Finally, in block 3 , the model RMSE $=-0.709+0.004 \times$ VRT was calculated $\left(R=0.503, R^{2}=0.253, p<0.05\right)$. In the young group, no significant model was calculated for any of the blocks.

Table 2

The multivariable linear regression analysis of each block in the acquisition phase for the elderly group.

$\begin{array}{llll}\text { RMSE of each block } & S E \beta(95 \% \mathrm{Cl}) & B & p \text {-Value }\end{array}$

Block1

\begin{tabular}{|llll} 
100g AGF task & $0.639(6.711-30.432)$ & 18.571 & 0.004 \\
VMS & - & - & 0.442 \\
$\mathrm{R}^{2}$ & 0.408 & \\
F & 11.018 & \\
Block2 & & - & 0.240 \\
100g AGF task & - & 0.003 & 0.037 \\
VMS & $0.495(0.000-0.005)$ & & \\
$\mathrm{R}^{2}$ & 0.245 & & \\
$\mathrm{~F}$ & 5.195 & &
\end{tabular}

Block3

$\begin{array}{llll}100 \mathrm{~g} \text { AGF task } & - & - & 0.631 \\ \text { VMS } & 0.503(0.000-0.007) & 0.004 & 0.033 \\ \mathrm{R}^{2} & 0.253 & & \\ \mathrm{~F} & 5.419 & & \end{array}$

Note: SE $\beta$ : standardised coefficient; Cl: confidence interval, RMSE: Root Mean Square Error, VMS: visual-motor speed

\section{Discussion}

The elderly have been reported to perform worse than young people in AGF tasks performed using visual information[1, 2]. However, the effect of the ability to properly adjust the grasping force without relying on other senses and the ability to reflect visual information in movement affect has not been investigated. In addition, it was not clear whether practice using concurrent visual feedback, which is effective for motor learning in the elderly, works effectively for learning the AGF task. Therefore, we investigated the effect of practice using concurrent visual feedback on learning the AGF task in two generations and investigated 
how VMS and the difference between the desired performance and the actual performance affect AGF in the elderly.

As a result of this study, the elderly group effectively enhanced motor learning of the AGF task by practising with concurrent visual feedback, as in the young group. Smitha et al.[21] reported that procedural learning was not significantly impaired in the elderly. A previous study reported that concurrent visual feedback was effective for motor learning in the elderly group in a coordinated task on both upper limbs. In other words, it shows the possibility of motor learning in the elderly and the effect of practice with concurrent visual feedback. However, although motor learning occurred in both groups, the elderly group could not reach the level of the young group. Certain reports establish that it is difficult for the elderly to learn the same performance as young people[22, 23]. Coats et al.[24] reported that the learning effects of practising with concurrent visual feedback on the visual coordination task with the upper limb were about half of that of younger people when compared with the elderly, resulting from age-related decline in visual motion perception. Thus, the results of this study show that concurrent visual feedback is effective in motor learning in the elderly, and it is difficult for the elderly to achieve the same performance as the young in the AGF task. In addition, the multivariable linear regression analysis of the RMSE of the retention test showed that age and pre-test performance affected the retention test performance. In addition, young people performed better on both VRT and 100g AGF tasks than the elderly. From these facts, the difference between the desired performance and the actual performance may become significant, and VMS decreases in the elderly. Furthermore, the learning effect for the elderly obtained through the practice using the concurrent visual feedback is smaller than that for young people.

Regression analysis of the performance of the main learning task during the acquisition phase revealed that the performance of the elderly group was influenced by the performance of the $100 \mathrm{~g}$ AGF task in the early stage of the practice and VRT in the latter stage. At the early stage of the acquisition phase, no significant difference was observed in the comparison between the groups of the $100 \mathrm{~g}$ AGF task, but the effect size was moderate, so it is possible that the RMSE of the 100g AGF task was larger in the elderly group than in the young group. Therefore, there may be a large difference between the desired exercise and exercise performed. Moreover, there is a possibility that the difference between the desired performance and actual performance in the elderly is larger when compared with the young. Vieluf et al. [25] reported that the error in isometric force adjustment when a pinch motion was performed without visually presenting a target value was larger in the elderly than in the young. This result is consistent with the results of the $100 \mathrm{~g} \mathrm{AGF}$ task in this study. Welford et al.[26] also stated that aging increases the level of noise in the motor system, which is a major cause of poor performance. This is considered as one of the reasons the AGF of the elderly is lower than the young. Moreover, the elderly group showed a larger VRT value than the young group. This result is consistent with those of many previous studies[16-18]. Furthermore, it was clarified that the larger the VRT, the lower the performance in the latter stage of practice in the elderly. Previous studies have reported that the elderly are more likely to generate motion noise when a minor force adjustment is required during force adjustment based on visual information[27]. It can be seen that the task using iWakka required fine adjustment of the force of $400 \mathrm{~g}$ or less using visual information, and this tendency was more remarkable. In addition, the elderly have taken 
longer to reach performance comparable to that of young people[4]. From these facts, it takes time for the elderly to correct the difference between desired performance and actual performance at the initial stage of the practice. Furthermore, the degree of improvement by the practice is small in the elderly with a slow visual-motor speed. Conversely, for young people, the degree of the $100 \mathrm{~g}$ AGF task and VRT before the practice was high, and it is considered that the performance during practice was immediately improved and led to learning.

This study has some limitations. First, in this study, it was clarified that the visual-motor speed and the difference between desired performance and the actual performance had effects on the performance during the practice, but these functions were not measured the day after that. It is necessary to confirm whether these functions are changed by practice with concurrent visual feedback and investigate whether this degree is related to the motor learning effect. In addition, in this study, we measured the difference between desired performance and actual performance, and VMS was predicted to be strongly related to the situation using concurrent visual feedback, but other functions also decline with ageing. Thus, it may be necessary to examine the effects of other functions, such as attentional and cognitive functions, which are likely to decline with ageing, on AGF.

Finally, Kaneno et al.[1] reported that the elderly had lower AGF than younger individuals in situations where concurrent visual feedback was used, as in the acquisition phase of this study. The authors state the need for an intervention regarding this ability. The results of this study showed that the difference between desired performance and actual performance and visual-motor speed was associated with a decrease in AGF. Therefore, intervention for these functions before the start of the practice of the learning task may lead to improvement of AGF and promotion of the motor learning effect.

\section{Conclusion}

In this study, we investigated whether the difference between desired performance and the actual performance and visual-motor speed was related to AGF in the elderly and investigated the effect of practice using concurrent visual feedback on AGF tasks. The results of this study showed that concurrent visual feedback worked effectively for motor learning of the AGF task in the elderly, and the degree was related to age and pre-practice performance. It was also found that in practice using concurrent visual feedback, the performance in the early stages was affected by the difference between desired performance and the actual performance and by VMS as the practice progressed. The results of this study provide new insights that facilitate the learning of AGF tasks in the elderly.

\section{Declarations}

\section{Data availability}

Data is available upon reasonable request to the corresponding author.

\section{Contributions}

Page 10/15 
R.Y. and K.A. designed the experiment, T.K. provided computational support for the experiment, R.Y. and J.Y. ran the experiment, R.Y. and K.Y. analyzed the results, all authors contributed to writing the article.

\section{Ethics declarations}

\section{Competing interests}

The authors declare no competing interests.

\section{References}

1. Kaneno, T. et al. A comparative study of adjustability of grasping force between young people and elderly individuals. Asian J. Occup. Ther, 15, 69-75 https://doi.org/10.11596/asiajot.15.69. (2019).

2. Kaneno, T. et al. Assessing the adjustability of grasping force using the iWakka in elderly individuals. J. Phys. Ther. Sci. 29, 2215-2219(2017). 10.1589/jpts.29.2215, Pubmed:29643609

3. Raz, N., Williamson, A., Gunning-Dixon, F., Head, D. \& Acker, J. D. Neuroanatomical and cognitive correlates of adult age differences in the acquisition of a perceptual-motor skill. Microsc. Res. Tech, 51, 85-93 https://doi.org/10.1002/1097-0029(20001001)51:1<85.. (2000). :AID-JEMT9>3.0.C0;2-0, Pubmed:11002356

4. Rodrigue, K. M., Kennedy, K. M. \& Raz, N. Ageing and longitudinal change in perceptual-motor skill acquisition in healthy adults. J. Gerontol. B Psychol. Sci. Soc. Sci. 60, P174-P181 (2005). 10.1093/geronb/60.4.p174, Pubmed:15980284

5. Wolpert, D. M., Diedrichsen, J. \& Flanagan, J. R. Principles of sensorimotor learning. Nat. Rev. Neurosci. 12, $739-751$ (2011). 10.1038/nrn3112, Pubmed:22033537

6. Holding, D. H. Principles of Training: Knowledge of Results 15-35 (Pergamon, 1965)

7. Smyth, M. M. Attention to visual feedback in motor learning. J. Mot. Behav, 10, 185-190 https://doi.org/10.1080/00222895.1978.10735152, Pubmed:15186980. (1978).

8. Vander Linden, D. W., Cauraugh, J. H. \& Greene, T. A. The effect of frequency of kinetic feedback on learning an isometric force production task in nondisabled subjects. Phys. Ther, 73, 79-87 https://doi.org/10.1093/ptj/73.2.79,. (1993). Pubmed:8421721

9. Schmidt, R. A. \& Wulf, G. Continuous concurrent feedback degrades skill learning: Implications for training and simulation. Hum. Factors 39, 509-525 (1997). 10.1518/001872097778667979, Pubmed:9473972

10. Huegel, J. C. \& O'Malley, M. K. (2010). Progressive haptic and visual guidance for training in a virtual dynamic task in Haptics Symposium IEEE 343-350

11. Wulf, G., Shea, C. H. \& Matschiner, S. Frequent feedback enhances complex motor skill learning. J. Mot. Behav. 30, 180-192 (1998). 10.1080/00222899809601335, Pubmed:20037033

12. Lee, M., Moseley, A. \& Refshauge, K. Effect of feedback on learning a vertebral joint mobilization skill. Phys. Ther. 70, 97-102; discussion 103 (1990). 10.1093/ptj/70.2.97, Pubmed:2296617 
13. Sigrist, R., Rauter, G., Riener, R. \& Wolf, P. Augmented visual, auditory, haptic, and multimodal feedback in motor learning: A review. Psychon. Bull. Rev, 20, 21-53 https://doi.org/10.3758/s13423012-0333-8,. (2013). Pubmed:23132605

14. Wishart, L. R., Lee, T. D., Cunningham, S. J. \& Murdoch, J. E. Age-related differences and the role of augmented visual feedback in learning a bimanual coordination pattern. Acta Psychol, 110 (02), 247-263 https://doi.org/10.1016/s0001-6918 (2002). )00036-7, Pubmed:12102108.

15. Baker, E. L. et al. A computer-based neurobehavioral evaluation system for occupational and environmental epidemiology: Methodology and validation studies. Neurobehav. Toxicol. Teratol, 7, 369-377 (1985). Pubmed:4058646.

16. Wilkinson, R. T. \& Allison, S. Age and simple reaction time: Decade differences for 5,325 subjects. J. Gerontol. 44, P29-P35 (1989). 10.1093/geronj/44.2.p29, Pubmed:2921473

17. Anstey, K. J., Dear, K., Christensen, H. \& Jorm, A. F. Biomarkers, health, lifestyle, and demographic variables as correlates of reaction time performance in early, middle, and late adulthood. Q. J. Exp. Psychol. A, 58, 5-21 (2005). 10.1080/02724980443000232, Pubmed:15881288.

18. Dykiert, D., Der, G., Starr, J. M. \& Deary, I. J. Age differences in intra-individual variability in simple and choice reaction time: Systematic review and meta-analysis. PLOS ONE 7, e45759 (2012). 10.1371/journal.pone.0045759, Pubmed:23071524

19. Krampe, R. T. Ageing, expertise and fine motor movement. Neurosci. Biobehav. Rev, 26, 769-776 (2002). 10.1016/s0149-7634(02)00064-7, Pubmed:12470688.

20. Cuthbertson, D. W., Bershad, E. M., Sangi-Haghpeykar, H. \& Cohen, H. S. Balance as a measurement of fatigue in postcall residents. Laryngoscope, 125, 337-341 https://doi.org/10.1002/lary.24792, Pubmed:24933004. (2015).

21. Smith, C. D. et al. Memories that last in old age: Motor skill learning and memory preservation. Neurobiol. Ageing 26, 883-890(2005). 10.1016/j.neurobiolaging.2004.08.014, Pubmed:15718047

22. Brown, R. M., Robertson, E. M. \& Press, D. Z. Sequence skill acquisition and off-line learning in normal ageing. PLOS ONE, 4, e6683 https://doi.org/10.1371/journal.pone.0006683, Pubmed:19690610. (2009).

23. Ehsani, F., Abdollahi, I., Bandpei, M. A. M., Zahiri, N. \& Jaberzadeh, S. Motor Learning and Movement Performance: Older versus Young Adults. Basic Clin. Neurosci, 6, 231-238 (2015).

24. Coats, R. O., Snapp-Childs, W., Wilson, A. D. \& Bingham, G. P. Perceptuomotor learning rate declines by half from 20s to 70/80s. Exp. Brain Res, 225, 7584 (2013).

25. Vieluf, S. et al. Dynamical signatures of isometric force control as a function of age, expertise, and task constraints. J. Neurophysiol. 118, 176-186(2017). 10.1152/jn.00691.2016, Pubmed:28356479

26. Welford, A. T. Signal, noise, performance, and age. Hum. Factors, 23, 97-109 (1981). 10.1177/001872088102300109, Pubmed:7228049.

27. Enoka, R. M. et al. Mechanisms that contribute to differences in motor performance between young and old adults. J. Electromyogr. Kinesiol, 13, 1-12 https://doi.org/10.1016/s1050-6411(02)00084-6,. (2003). Pubmed: 12488083 
Figures
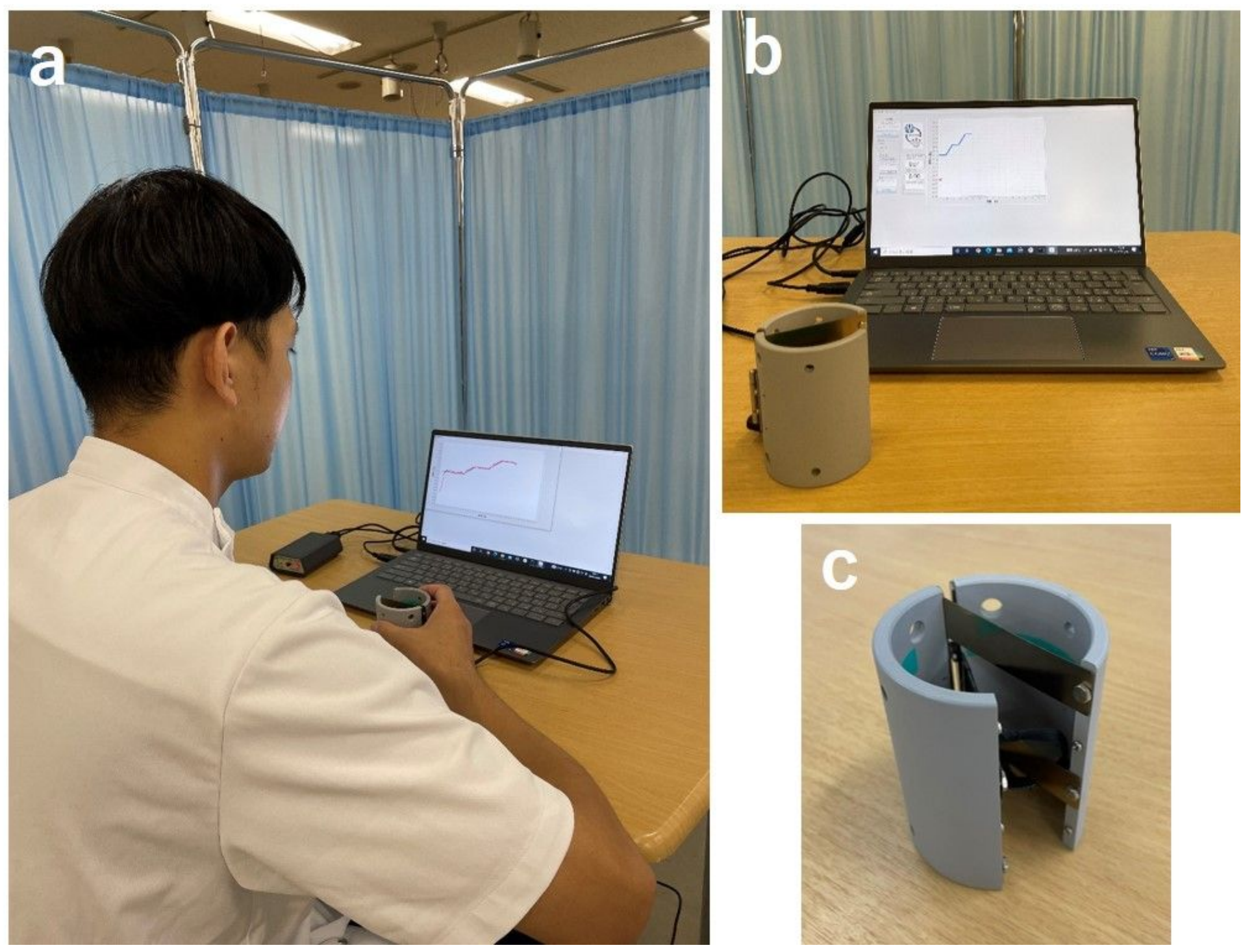

\section{Figure 1}

iWakka a. state when using iWakka, b. iWakka and monitor/computer displaying feedback, c. iWakka 


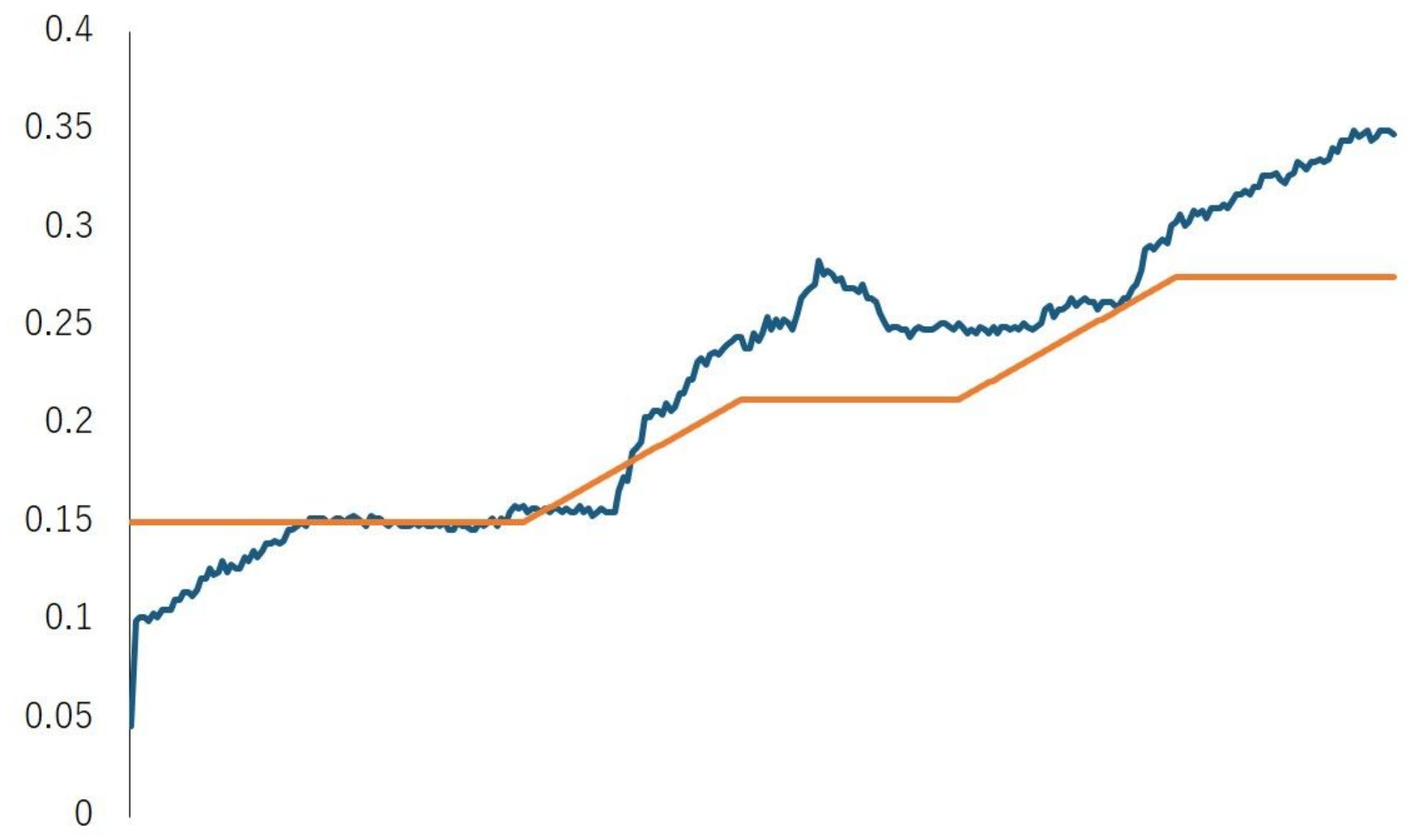

Figure 2

Waveforms of the main learning task and feedback screen

Edinburgh Handedness Inventory

The 100g AGF Task

(difference between desired performance and actual performance) Visual Reaction Time

(Visual Motor Speed)

The Learning Task

Pretest

Acquisition Phase

24 hours after

Retention Test

Figure 3 
Procedure In the $100 \mathrm{~g}$ AGF task, 5 trials were performed without giving feedback. The number of Visual Reaction Time measurements was 5 trials. Regarding the main learning task, the pretest was conducted in 4 trials without feedback. The acquisition phase consisted of 4 trials $\times 3$ blocks for a total of 12 trials, and concurrent feedback was given during the performance. The retention test was conducted about 24 hours after the acquisition phase in the same manner as the pretest.

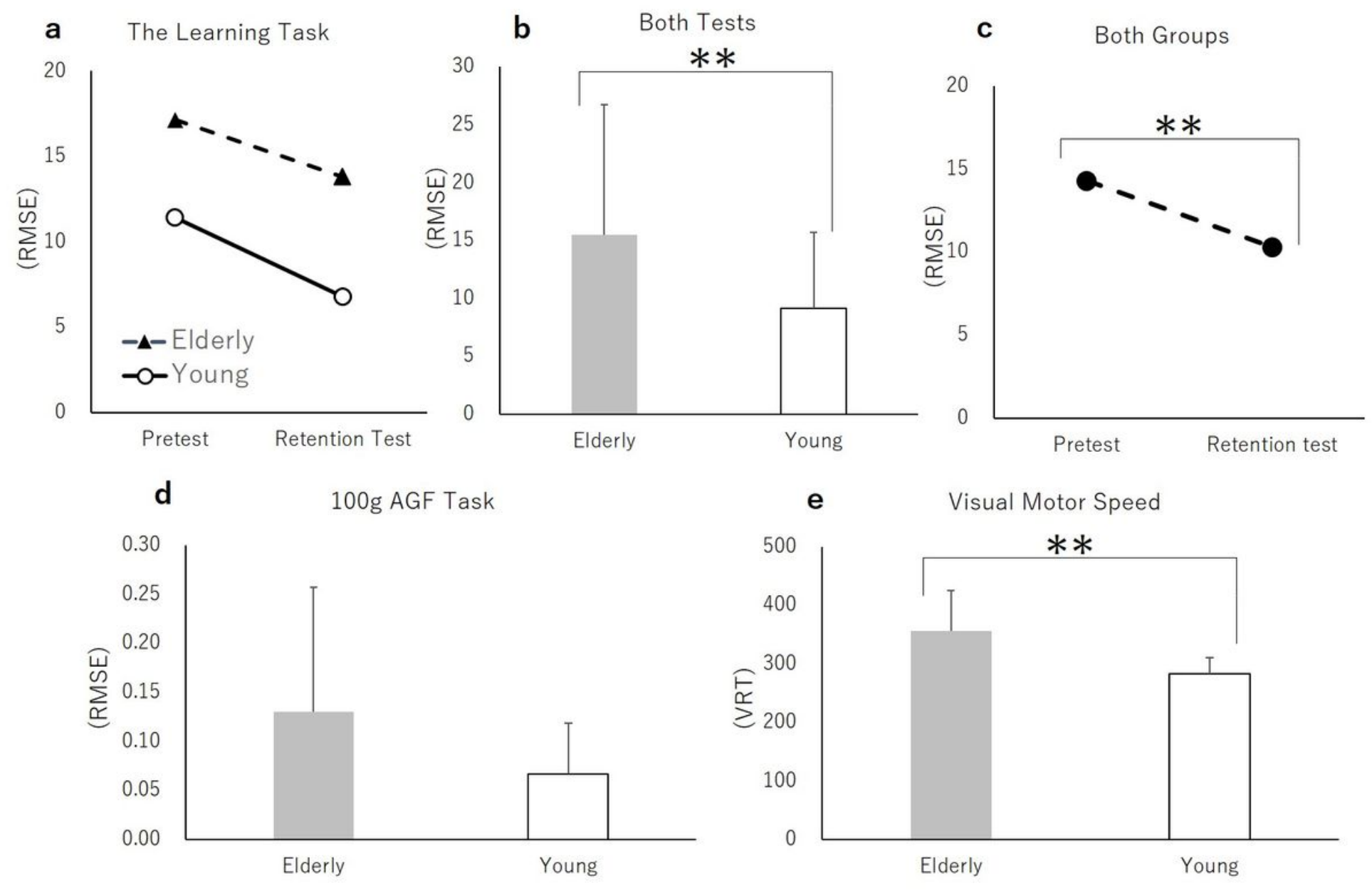

\section{Figure 4}

Results of each measurement item a. RMSE of the pretest and the retention test (the main learning task), b. Comparison between groups of both tests (the main learning task), c. Comparison of pretest and retention test for all participants (the main learning task), d. Comparison of the $100 \mathrm{~g}$ AGF task, e. comparison of visual-motor speed, $\left({ }^{*} p<0.05,{ }^{* *} p<0.01\right)$. 